\title{
New Methods for Selecting Stallion Spermatozoa for Assisted Reproduction
}

\author{
Silvia Colleoni, Irina Lagutina, Giovanna Lazzari, Heriberto Rodriguez-Martinez, \\ Cesare Galli and Jane M Morrell
}

\section{Linköping University Post Print}

N.B.: When citing this work, cite the original article.

Original Publication:

Silvia Colleoni, Irina Lagutina, Giovanna Lazzari, Heriberto Rodriguez-Martinez, Cesare Galli and Jane M Morrell, New Methods for Selecting Stallion Spermatozoa for Assisted Reproduction, 2011, Journal of Equine Veterinary Science, (31), 9, 536-541.

http://dx.doi.org/10.1016/j.jevs.2011.03.009

Copyright: WB Saunders http://www.elsevier.com/

Postprint available at: Linköping University Electronic Press

http://urn.kb.se/resolve?urn=urn:nbn:se:liu:diva-71386 


\title{
New methods for selecting stallion spermatozoa for assisted reproduction.
}

\section{Colleoni $\mathrm{S}^{\mathbf{1}}$, Lagutina $\mathbf{I}^{\mathbf{1}}$, Lazzari $\mathbf{G}^{\mathbf{1}}$, Rodriguez-Martinez $\mathbf{H}^{\mathbf{2}}$, Galli $\mathrm{C}^{\mathbf{1 3}}$, JM Morrell $^{4} *$}

\author{
${ }^{1}$ AVANTEA, Laboratorio di Tecnologie della Riproduzione, Cremona, Italy \\ ${ }^{2}$ Department of Clinical and Experimental Medicine, Linköping University, Linköping, \\ Sweden \\ ${ }^{3}$ Dipartimento Clinico Veterinario, Università di Bologna, Bologna, Italy \\ ${ }^{4}$ Division of Reproduction, Swedish University of Agricultural Sciences, Uppsala,
}

Sweden

\begin{abstract}
Improved sperm selection techniques are needed to increase the efficiency of equineassisted reproduction. Single layer centrifugation (SLC) of spermatozoa has been shown to improve the quality of stallion sperm samples. In this study, the functionality of selected stallion spermatozoa was tested by intracytoplasmic sperm injection of equine oocytes after selection by SLC through Androcoll-E or by discontinuous density gradient centrifugation (DGC) through Redigrad and Tyrode's medium with added albumin, lactate, and pyruvate. The mean cleavage rates of the injected oocytes from SLC- and DGC-selected spermatozoa were $67 \%$ and $66 \%$, respectively, whereas the proportion of blastocysts developing from cleaved oocytes was $28 \%$ and $22 \%$, respectively ( $\mathrm{P}>.05$, not significant). An incidental finding was that there was a tendency for SLC-selected spermatozoa to have a higher percentage of spermatozoa with normal morphology than DGC $(70 \% \pm 22 \%$ vs. $58 \% \pm 38 \%)$ and for more blastocysts to be obtained from subfertile ejaculates $(21$ [19.6\%] vs. 15 [14.4\%], respectively). In further experiments, stallion spermatozoa bound to hyaluronan, although binding may depend on the semen extender and sperm treatment as well as incubation time. In conclusion, SLC-selected stallion spermatozoa function normally when injected into oocytes. SLC may potentially be better than DGC at selecting spermatozoa from subfertile ejaculates, but this effect needs rigorous investigation with a much larger sample size. Use of the hyaluronanbinding assay for assessing the potential fertility of stallion spermatozoa may be useful but requires further evaluation.
\end{abstract}

*Corresponding author: Jane M. Morrell, BVetMed, PhD, Clinical Sciences, SLU, Box 7054, SE-75007 Uppsala, Sweden

Key words: ICSI, Stallion spermatozoa, Hyaluronan binding, Androcoll-E, Single layer centrifugation 


\section{Introduction}

In most of the farm animal species, sire selection is made on the basis of their fertility. This aspect is not usually considered for stallions because top sires are always chosen for their excellence in sportive performances or for their genetic background. For this reason, fertility and freezability of stallion sperm presents a highly variable, even since the development of efficient freezing protocols (Loomis and Graham, 2008). To date, for some stallions with low quality semen, the only way to obtain offspring is the selection of most fertile spermatozoa associated with low dose insemination AI or in vitro embryo production.

A new method for selecting the most robust spermatozoa from animal ejaculates has been developed recently at SLU (Morrell and Rodriguez-Martinez, 2009). The new method involves passage of spermatozoa through a single layer of glycidoxypropyltrimethoxysilane-coated silica colloid (Androcoll ${ }^{\mathrm{TM}}$ ). This technique, known as Single Layer Centrifugation (SLC), is simpler and quicker to perform than conventional density gradient centrifugation (DGC). Comparisons of the two techniques have been carried out using species-specific formulations of Androcoll ${ }^{\mathrm{TM}}$ with stallion and boar semen. The two techniques select the most robust spermatozoa, in terms of motility, morphology, viability and chromatin integrity, from the rest of the ejaculate (Morrell et al, 2009a, b) and SLC is more user-friendly and can be scaled-up to process large volumes of semen (Morrell et al, in press). In addition, the fertilizing ability of selected spermatozoa prepared by DGC and SLC was not different in a bovine in vitro fertilization (IVF) study (Thys et al, 2009). In the bovine species, where variations in fertility between bulls is lower than among stallions, no differences in sperm quality could be detected between the two methods in the above studies and it was concluded that they were similarly effective in selecting the most robust spermatozoa. Nevertheless, in horses, the effectiveness of different separation techniques still requires investigation.

Equine artificial insemination (AI) trials are notoriously difficult to conduct, partly because of the large numbers of animals required (Amann, 2005) and also because of the need for controls, in this case non-SLC-selected sperm doses, which are unacceptable to the owners of mares if the ejaculates originate from sub-fertile stallions. Although isolated cases of AI with SLC-selected stallion spermatozoa have been highly successful (Morrell et al, unpublished data), more data are required concerning the fertilizing ability of these selected spermatozoa. Ideally, IVF would be an effective way of obtaining fertilization data quickly. However, the methodology for equine IVF is considered to be unsuccessful, owing to problems of maturation of the zona pellucida (ZP) in vitro, and with only two foals produced using the technique over the past two decades, both being derived from in vivo matured oocytes (Palmer et al. 1991, Bezard et al. 1992). In the meantime, other techniques developed for human assisted reproduction have been utilized to produce equine embryos, including oocyte transfer (Carnevale, 2004) and intracytoplasmic sperm injection (ICSI) (Galli et al., 2002). Recently, two studies (Roasa et al., 2007; McPartlin et al., 2009) have re-evaluated the IVF technique, focusing on sperm preparation. These authors demonstrated that factors such as variability between ejaculates, composition of media, freezing extender and capacitation process affect fertilization rate. Their studies suggest that sperm treatment and preparation are key factors for embryo production by IVF, although the technique remains limited in efficiency. In contrast, application of ICSI to equine oocytes is 
relatively successful, provided that a motile spermatozoon is selected for injection (Lazzari et al, 2002). However, when spermatozoa from stallions of low fertility were used, lower cleavage rates and lower development was achieved compared to three control stallions of proven fertility (Colleoni et al, 2009). In the light of these results a more efficient selection technique for the most fertile spermatozoa would be an interesting option to improve the efficiency of embryo production.

One possibility recently developed in human ART is the use of dishes coated with hyaluronon (synthetic hyaluronic acid) to facilitate selecting spermatozoa that are capable of fertilization for ICSI. Previous studies have shown that fertile human spermatozoa bind to glass slides coated with hyaluronic acid (Worrilow et al, 2003; Huszar et al, 2004; Roudebush et al, 2004). A binding assay (HBA; Medicult, Denmark) is commercially available whereby the binding of intact spermatozoa to glass slides coated with recombinant hyaluronic acid (hyaluronan) is thought to be indicative of the potential fertility of a human semen sample in subsequent IVF. However, it is not known whether stallion spermatozoa have similar receptors that are capable of binding to hyaluronan, nor whether such binding could be indicative of fertility in AI, nor if equine spermatozoa express a surface-associated hyaluronidase involved in the sperm-oocyte interaction (Meyers, 2001). Recently the same company has produced PICSI dishes - dishes coated with hyaluronan that allow immobilized spermatozoa to be selected for ICSI.

The objectives of the current study were three-fold: (i) to test the fertility of SLCselected spermatozoa by intracytoplasmic sperm injection (ICSI) and the subsequent development of the injected oocytes to the blastocyst stage; (ii) investigation of the use of the HBA for stallion spermatozoa and its link with potential fertility; and (iii) evaluation of the PICSI dishes as an aid to selecting stallion spermatozoa for ICSI. Since the laboratory where the ICSI was performed usually prepared spermatozoa using DGC, the ICSI trial also involved an indirect comparison of SLC and DGC, albeit with different colloid formulations.

\section{Materials and Methods \\ Oocyte maturation}

Equine cumulus-oocyte complexes (COCs) were recovered in the laboratory from ovaries collected at a local abbatoir by manually scraping and washing all follicles of $0.5-2.5 \mathrm{~cm}$ in diameter. Recovered oocytes were washed in TCM199 hepes-buffered medium, immediately transferred to DMEM-F12-based medium for maturation, and were cultured for $24 \mathrm{~h}$ at $38.5^{\circ} \mathrm{C}$ in a humidified atmosphere containing $5 \% \mathrm{CO}_{2}$ (Galli et al, 2007). After maturation, the cumulus cells were removed after a short incubation in hepesbuffered SOF (H-SOF) containing hyaluronidase (Lazzari et al, 2002) by gentle mouth pipetting in $\mathrm{H}-\mathrm{SOF}$ supplemented with $10 \%$ fetal bovine serum. Oocytes were returned to the maturation medium and those that showed first polar body extrusion were destined for ICSI.

\section{Sperm preparation for ICSI}

Straws of frozen semen from 3 stallions were available. One of these stallions (stallion X) was considered to be of normal fertility on the basis of field and in vitro results; his semen was used as a system control in all ICSI experiments performed in the laboratory. The other stallions ( $\mathrm{V}$ and $\mathrm{W}$ ) were classified as having poor fertility in AI based on field 
results. Straws were thawed by plunging them into water at room temperature $\left(25^{\circ} \mathrm{C}\right)$ for $30 \mathrm{~s}$.

In addition, one ejaculate of fresh semen extended 1:1 in INRA96 was available from a fourth stallion, stallion Y, also known to be of poor fertility.

Aliquots of thawed semen or the fresh ejaculate were prepared by SLC with Androcoll-E (SLU, Uppsala, Sweden) or by a density gradient consisting of layers of colloid prepared from Redigrad and TALP (45 and 90\%), which was the usual method in the laboratory.

SLC: up to $0.5 \mathrm{~mL}$ thawed semen were layered on top of $4 \mathrm{~mL}$ Androcoll ${ }^{\mathrm{TM}}$-E in a conical centrifuge tube and centrifuged at $300 \mathrm{~g}$ for $20 \mathrm{~min}$ (Thys et al, 2009). The resulting sperm pellet was washed in TALP (4 ml) by centrifuging at $500 \mathrm{~g}$ for $10 \mathrm{~min}$.

DGC: Redigrad and TALP were mixed to form a $90 \%$ colloid. An aliquot of the $90 \%$ colloid was mixed 1:1 (v/v) with TALP to form $45 \%$ colloid. The $45 \%$ colloid $(2 \mathrm{~mL})$ was layered on top of the $90 \%$ colloid $(2 \mathrm{~mL}$ ), and thawed semen (up to $0.5 \mathrm{ml}$ ) was pipetted on top. After centrifugation at $700 \mathrm{~g}$ for $40 \mathrm{~min}$, the resulting sperm pellet was resuspended in $4 \mathrm{ml}$ TALP and washed by centrifugation at $500 \mathrm{~g}$ for $10 \mathrm{~min}$. Sperm pellets obtained with both methods were resuspended in SOF-IVF medium to a concentration of 4 million sperm/ml and subsequently diluted 1:1 in PVP $12 \%$ in PBS prior to ICSI.

ICSI

ICSI was performed using a piezo-drill to breach the ZP(Galli et al, 2007). For the injection only motile spermatozoa with apparently normal morphology were chosen, immobilized with two or three piezo-drills and injected into the cytoplasm. Following ICSI, the injected oocytes were cultured in drops of $20 \mu \mathrm{L}$ under mineral oil in SOF medium supplemented with MEM amino acids and bSA (Lazzari et al, 2002). The medium was partially changed $(50 \%)$ at day 4 and day 6 post ICSI. Blastocyst formation was evaluated from day 6 to day 9 after ICSI. The following data were recorded: number of oocytes injected, cleavage rate, number of blastocysts at day 7 , total number of blastocysts, development of blastocysts from cleaved, injected oocytes, and development of blastocysts from injected oocytes.

\section{Sperm preparation for $H B A$}

Semen was collected from 11 stallions at a commercial stud (Flyinge AB, Flyinge, Sweden). The ejaculates were immediately extended 1:1 with INRA96 (IMV, l'Aigle, France), processed for AI doses in the usual manner and sent off to other studs for AI. An aliquot $(0.5 \mathrm{~mL})$ was removed from the processed semen and stored overnight at $6^{\circ} \mathrm{C}$. On the following day, these aliquots were further extended in phosphate buffered saline (PBS) to a concentration of approximately $2 \times 10^{6} / \mathrm{mL}$ and a $10 \mu \mathrm{L}$ drop was applied to the hyaluronan-coated glass slide (HBA; Medicult, Denmark). After incubation at $22^{\circ} \mathrm{C}$ for 15 minutes, sperm motility on the slides was analysed using the Qualisperm ${ }^{\mathrm{TM}}$ Motility Analyser (Biophos, Switzerland), to assess the proportion of the sperm population that were bound to the hyaluronan, i.e. showing vigorous tail movements without any progressive motility. 


\section{PICSI dishes}

Semen from the same stallions used for the SLC/DGC experiment was used for the evaluation of the PICSI dishes. An additional stallion of proven fertility was tested in some PICSI trials. Aliquots of the SLC-selected sperm suspensions were added to the PICSI dishes at $25^{\circ} \mathrm{C}$ according to the manufacturer's instructions. After a minimum of 10 minutes incubation, individual spermatozoa that had bound to the hyaluronan coating were removed, transferred into a drop containing PVP 12\% diluted 1:1 with SOF-IVF medium, examined for morphology, immobilized with two or three piezo-drills and injected into the oocytes.

\section{Sperm Morphology}

Briefly, aliquots of the frozen-thawed semen and of the SLC and DGC samples were fixed in formol-saline for the evaluation of 200 spermatozoa/preparation in wet smears (x1000) and to assess sperm head shape in 500 spermatozoa after staining with Williams' stain (Lagerlöf, 1934). For the DGC and SLC samples prepared from frozen-thawed semen for ICSI, there was occasionally insufficient material to enable 500 spermatozoa to be evaluated. The mean proportion of morphologically normal spermatozoa was estimated as the remaining proportion left after deducting the total abnormal spermatozoa. Spermatozoa with distal cytoplasmic droplets were included as normal. Data were also available from a previous comparison of SLC and DGC using stallion ejaculates $(n=38)$. The morphological examinations were performed by skilled staff from the sperm laboratory at the Swedish University of Agricultural Sciences, which acts as a reference laboratory for Sweden.

\section{Sperm chromatin structure assay}

The method used was originally described by Evenson et al, (2002) with subsequent modification (Johannisson et al, 2009). Briefly, aliquots $(100 \mu \mathrm{L})$ of the sperm samples were diluted 1:1 (v/v) in TNE buffer (Evenson \& Jost, 2006), snap-frozen in liquid nitrogen and stored at $-80^{\circ} \mathrm{C}$ until required for staining with acridine orange and subsequent flow cytometric analysis. The parameters measured were the ratio of single stranded to double stranded DNA [DFI (DNA fragmentation index, \%)], its standard deviation (SD_DFI) and the measure of single stranded DNA (mean_DFI).

\section{Statistical analysis}

Data analysis was performed using the statistical software package Statistical Analysis Systems software (SAS version 8, SAS Institute Inc, 1998; Cary, NC, USA). The distribution of variables from the statistical model was tested using the UNIVARIANCE procedure option NORMAL. Variation between stallions was analyzed using PROC GLM and Least Squares Means for each treatment, where the statistical model included the fixed effect of stallion. The differences between cleavage and blastocyst development in the PICSI trial were analyzed using Student's t test. In all cases, significance was set at $\mathrm{P}<0.05$ level.

\section{Results}


Experiment 1: comparison of SLC with Androcoll ${ }^{\mathrm{TM}}-E$ and $D G C$.

The ICSI results for the two methods of sperm separation for 4 stallions are shown in Table 1. Since semen from stallion Y was available on only one occasion, the results from this stallion have not been included in the statistical analysis. For the 3 stallions, V, W and X, a total of 135 and 131 oocytes were available for ICSI with SLC and DGCselected spermatozoa respectively. The mean cleavage rates of the injected oocytes were $66.7 \%$ and $66.4 \%$ respectively, whilst the proportion of blastocysts developing from cleaved oocytes was $28 \%$ and $22 \%$ respectively, and from injected oocytes was $19 \%$ and $15 \%$ respectively. There was no statistical difference between the two centrifugation methods, or between stallions $\mathrm{X}, \mathrm{V}$ and $\mathrm{W}$, despite two stallions ( $\mathrm{V}$ and $\mathrm{W}$ ) being of known sub-fertility.

The morphology results of the sperm preparations used in the ICSI trial showed that the two methods were very similar in their ability to select spermatozoa with normal morphology (means $63 \pm 30 \%$ and 59 $\pm 27 \%$ normal morphology for SLC and DGC respectively) but differed slightly in their ability to remove spermatozoa with morphological defects (Table 2). There were fewer spermatozoa with pathological heads (64.8 vs. $72 \%)$, midpiece defects $(23.5 \%$ vs. $42.5 \%)$ or with nuclear vacuoles (3\% vs. $11 \%$ ) in the SLC samples than in the DGC samples.

Figure 1 contains the SCSA results from SLC- and DGC-samples. Again, the SLC preparations showed a lower proportion of spermatozoa with damaged DNA than the DGC samples. However, whereas stallions $\mathrm{W}$ and $\mathrm{X}$ showed lower \%DFI in the selected samples than in the uncentrifuged samples, stallion $\mathrm{V}$ had a much higher \%DFI in the centrifuged preparations than in the uncentrifuged samples.

Experiment 2: evaluation of HBA slides used with stallion spermatozoa.

Binding to hyaluronan occurred but varied largely between stallions (range 11-24\%, median 14.5\%) (Figure 2).

Experiment 3: evaluation of PICSI dishes for selecting stallion spermatozoa for ICSI.

Binding to HA drops was highly variable between stallions. Stallion V showed no binding and was excluded from the experiment. Stallion W showed a very low level of binding and often there were insufficient bound spermatozoa to inject all the oocytes allocated for the injection. Stallion X (used in the previous SLC/DGC experiment as a control) showed affinity for HA. However, the tail movement was vigorous and the sperm were not bound stably, making sperm pick-up very difficult. Only spermatozoa from the additional control stallion were bound correctly, although the rate of binding was variable between straws.

In spite of these differences, the outcome of the ICSI was similar for all the three stallions (Table 3 ). When the bound spermatozoa were selected for ICSI, the cleavage rate was significantly less $(\mathrm{p}<0,05)$ than for the control (SLC only) spermatozoa ( $47 \%$ vs. $70 \%$ ), although a significantly higher proportion of the cleaved embryos developed to blastocysts for the hyaluronan-bound spermatozoa than for the control spermatozoa (35\% vs. 20\%). Nevertheless the total number of blastocysts developing from the injected oocytes was not different between the two groups.

\section{Discussion}


The experiment was designed to test the functionality of SLC-selected spermatozoa by examining the cleavage and further development of equine oocytes injected with SLC-selected stallion spermatozoa. The results showed that SLC-selected spermatozoa are capable of triggering cleavage and development to the blastocyst stage after injection into homologous oocytes. Furthermore, there was a trend for higher cleavage rates and for more blastocysts to be produced for the SLC-selected spermatozoa than for DGC-selected spermatozoa from stallions that were suspected of being subfertile. This result is interesting and warrants further investigation with a larger sample size. Previous results from the same laboratory for both experimental groups of oocytes and commercial OPU-collected oocytes had not shown any difference between fertile and sub-fertile stallions in the developmental competence of ICSI-treated oocytes, provided that motile spermatozoa were selected for injection (Lazzari et al, 2002; Colleoni et al, 2007). However, more recently, when the analysis was widened to include sperm preparations from three infertile stallions used for ICSI of experimental oocytes collected from abbatoir ovaries, lower cleavage rates were found for two stallions and lower development was achieved for all three stallions compared to three different control stallions of proven fertility (Colleoni et al, 2009).

No statistical difference was identified in the ability of the SLC and DGC to select morphologically normal spermatozoa in the ICSI study, although it should be noted that only one aliquot was tested for each stallion. However, there were approximately $20 \%$ fewer spermatozoa in the SLC samples than in the DGC samples (data not shown), perhaps indicating that the selection was more pronounced when using the SLC method. In a previous study using fresh sperm samples from fertile stallions, some differences were observed in the ability of the two methods to remove certain abnormalities, with a trend for SLC to be slightly better than DGC in removing the abnormal spermatozoa, for example those with bent tails or pear-shaped heads ( $\mathrm{P}<0.001$ for both defects) (Morrell et al, submitted). However, the benefit of a greater selection ability of SLC over DGC when preparing spermatozoa for ICSI would be if more spermatozoa with nuclear vacuoles were removed by SLC than by DGC, since it is not possible to detect this serious defect in living spermatozoa by visual examination with the microscope when choosing the spermatozoa for injection. Other morphological abnormalities can be detected in living spermatozoa by a trained operator and hence these spermatozoa are avoided when choosing each spermatozoon for injection. The cleavage rates and development to blastocyst stage for DCG- prepared spermatozoa were consistent for the current experiment and for previous work using semen from stallions $\mathrm{X}$ and $\mathrm{V}$.

For all of the stallions, SLC was slightly better than DGC at selecting spermatozoa with normal DNA. However, for one stallion (V), DFI was far higher in the centrifuged samples than in the uncentrifuged samples. This result was surprising and is in contrast to other results, both with fresh sperm samples (Morrell et al, 2009c) and with frozen sperm samples (Macias et al, 2009). The reason for this high DFI is unknown. For the infertile stallions, $50 \%$ more blastocysts resulted from the frozen-thawed SLC sperm preparations from the infertile stallions than from the DGC sperm preparations (21 and 15 respectively), suggesting that SLC may be the method of choice when dealing with ejaculates from such stallions. These results are interesting and warrant further investigation. 
One explanation for the slightly better performance of SLC-selected spermatozoa in the further development of injected oocytes is that SLC may be better than DGC at selecting spermatozoa with normal, intact chromatin. It is not possible to detect chromatin damage visually in living spermatozoa, hence the chance of selecting a spermatozoon with damaged chromatin for ICSI is elevated if their presence in the sperm sample increases. It is known that fertilization by spermatozoa with damaged chromatin in IVF and ICSI leads to failure of subsequent development at some stage (Donnelly et al, 2000). Although the oocyte has the capability to repair some DNA-damage, extensively damaged sperm DNA may overcome this mechanism, resulting in a low rate of embryonic development (Ahmadi and $\mathrm{Ng}, 1999$ ). It has been established that the DNA fragmentation index (DFI) value of an ejaculate is strongly linked to its potential fertility for several species (including the stallion), with thresholds of $0-15 \%, 16-29 \%$ and $\geq 30 \%$ relating to high, moderate and low fertility potential respectively (Evenson \& Jost, 2000). Furthermore, significant relationships between specific morphological abnormalities and DFI have been established, including nuclear vacuoles and midpiece defect (Morrell et al, 2008). This aspect warrants investigation.

Although stored stallion spermatozoa did bind to the hyaluronan-coated slides, there was less binding than had been reported previously for human spermatozoa (2889\%) (Hong $\mathrm{Yi}$ et al, 2006). It is not known whether the receptors on stallion spermatozoa act in the same way as those on human spermatozoa. Alternatively, the extender used for maintaining the stallion spermatozoa during storage may have inhibited binding to the hyaluronan, even though PBS was subsequently added to the samples to reduce the sperm concentration to $2 \times 10^{6} / \mathrm{mL}$. It is intended to repeat the assay using semen extended only with PBS to see whether more binding to hyaluronan is achieved in this way. Moreover, it is claimed that with human spermatozoa, the binding to hyaluronan is predictive of success in IVF. However, AI of semen doses from the ejaculates used for the HBA in the study reported here gave a pregnancy rate of $88 \%$; without any correlation between low binding and non-pregnancy or, conversely, high binding and pregnancy. This might indicate that other factors also play a role in fertility after AI in horses, or that this selection system requires further development regarding horse spermatozoa, in terms of binding capacity.

The low binding of frozen-thawed stallion spermatozoa in the PICSI dishes confirms the observations with fresh (non-frozen), stored stallion spermatozoa binding to HBA slides. It was surprising that the cleavage rate after ICSI should have been much lower with the PICSI-selected spermatozoa than with the SLC-only controls, and also that the development rate of the cleaved oocytes should have been so much higher. There is no obvious explanation for these observations at present. However, in a previous study where boar spermatozoa that bound to hyaluronic acid drops were used for ICSI (ICSIHABS), significantly lower rates of chromosomal abnormality were seen in the ICSIHABS embryos than from control ICSI, despite the blastocysts rates being the same (Park et al, 2005). The authors concluded that ICSI-HABS was a superior method than ICSI for the production of porcine embryos with normal chromosomal complement (Park et al, 2005). The chromosomal composition of the embryos produced in the current study was not examined, although it can be speculated that the higher blastocyst development rate shown for the PICSI-group suggests that similar factors were involved here as for the porcine study. 
It was interesting to note that the cleavage rate and development to blastocyst stage for SLC-selected spermatozoa was the same in both the first experiment and the PICSI experiment, indicating the repeatability of the results when using the SLC technique

In conclusion, stallion spermatozoa subjected to SLC-selection on Androcoll-E are able to "fertilize" oocytes in ICSI, and the injected oocytes are capable of development to the blastocyst stage. SLC with Androcoll ${ }^{\mathrm{TM}}-\mathrm{E}$ may have an advantage over DGC in selecting normal spermatozoa from ejaculates from sub-fertile stallions. Use of the HBA and PICSI dishes for stallion spermatozoa requires further investigation to establish the usefulness of these techniques for equine biotechnologies.

\section{Acknowledgments}

This project was sponsored by a travel grant from the Nils Lagerlöf Foundation, Royal Academy for Forestry and Agriculture, Sweden, and by the Foundation for Equine Research, Sweden, Regione Lombardia, Italy, and Uterofert and Embryo-Genotyping projects. 


\section{References}

Ahmadi A, Ng SC. 1999 Fertilizing ability of DNA-damaged spermatozoa. J Expt. Zoology 284, 696-704.

Amann RP. 2005 Weaknesses in reports of "fertility" for horses and other species. Theriogenology 63, 698-715.

Bezard J. 1992. In vitro fertilization in the mare. In: Proceedings of the International Scientific Conference on Biotechnics in Horse Reproduction. Agricultural University of Cracow, Poland, Abstract 12.

Carnevale EM. 2004. Oocyte transfer and gamete intrafallopian transfer in the mare. Anim. Reprod. Sci. 82/83, 617-624, Review.

Colleoni S, Barbacini S, Necchi D, Duchi R, Lazzari G, Galli C. 2007 Application of ovum pick-up, intracytoplasmic sperm injection and embryo culture in equine practice. AAEP proceedings 554-559.

Colleoni S, Duchi R, Barbacini S, Necchi D, Mari G, Spinaci M, Lazzari G, Galli C 2009. Practical applications of OPU, ICSI and IVC in equine reproduction. Reproduction in Domestic Animals 44, Suppl.3, W2.2.

Donelly D, McClure N, Trinder S, Lewis SEM. 2000 Spermatozoal DNA integrity: correlation with fertilization and pregnancy rates in IVF and ICSI. Human Reproduction $15,1552-1561$.

Evenson, DP., Darzynkiewicz, Z. \& Melamed, M.R., 1980. Relation of mammalian sperm chromatin heterogeneity to fertility. Science 210, 1131-1133.

Evenson D \& Jost L. 2000 Sperm chromatin structure assay is useful for fertility assessment. Methods in Cell Science 22, 169-189.

Galli C, Crotti G, Turini P, Duchi R, Mari G, Zavaglia G, Duchamp G, Daels P, Lazzari G. 2002. Frozen-thawed embryos produced by ovum pickup of immature oocytes and ICSI are capable to establish pregnancy in the horse. Theriogenology 58, 705-08.

Galli C, Colleoni S, Duchi R, Lagutina I and Lazzari G. (2007) Developmental competence of equine oocytes and embryos obtained by in vitro procedures ranging from in vitro maturation and ICSI to embryo culture, cryopreservation and somatic cell nuclear transfer. Animal Reproduciton Science 98, 39-55.

Hong Ye, Guo-ning Huang, Yang Gao \& De Yi Liu (2006) Relationship between human sperm-hyaluronan binding assay and fertilization rate in conventional in vitro fertilization. Human Reproduction 21, 1545-1550. 
Huszar G, Ozenci C, Cayli, S, Zavaczki Z, Hansch E, Vigue, L. (2003) Hyaluronan acid binding by human sperm indciates cellular maturity, viability and unreacted acrosomal status. Fertil Steril 79, 1616-1624.

Johannisson, A., Morrell, J.M., Thorén, J, Jonsson, M, Dalin, A-M, Rodriguez-Martinez, H. (2009) Colloidal centrifugation with Androcoll-ETM prolongs stallion sperm motility, viability and chromatin integrity. Anim Reprod Sci. 116, 119-128.

Lagerlöf N. 1934 Morphological studies on the change in sperm structure and in the testes of bulls with decreased or abolished fertility. Acta Pathol Microbiol Scand Suppl 19, 254-267.

Lazzari G, Wrenzycki C, Herrmann D, Duchi R, Kruip T, Niemann H, Galli C (2002) Cellular and molecular deviations in bovine in vitro-produced embryso are related to the large offspring syndrome. Biol Reprod 67, 767-775.

Lazzari G, Crotti G, Turini P, Duchi R, Mari G, Zavaglia G, Barbacini S, Galli C. 2002. Equine embryos at the compacted morula and blastocyst stage can be obtained by intracytoplasmic sperm injection (ICSI) of in vitro matures oocytes with frozen-thawed spermatozoa of different fertilities. Theriogenology 58, 709-712.

Loomis PR, Graham JK. (2007). Commercial semen freezing: individual male variation in cryosurvival and the response of stallion sperm to customized freezing protocols. Anim. Reprod. Sci. 105, 119-128

McPartlin LA, Suarez SS, Czaya CA, Hiinrichs K, Bedford-Guaus SJ. 2009 Hyperactivation of stallion sperm is required for successful in vitro fertilization of equine oocytes. Biol reproduction DOI:10.1095/biolreprod.108.074880

Macias GB, Fernandez-Gonzalez L., Morrell J., Ortega Ferrusola C., Tapia JA, Rodriguez-Martinez H, Pena FJ. (2009) Single layer centrifugation through colloid positively modifies the sperm sub-population structure of frozen-thawed stallion spermatozoa. Anim Reprod Sci 114, 193-202.

Meyers SA, 2001. Equine sperm-oocyte interaction: the role of sperm surface hyaluronidase. Anim. Reprod. Sci. 68, 291-303.

Morrell, J.M., Johannisson, A., Dalin, A-M., Hammar, L., Sandebert, T, \& RodriguezMartinez, H. 2008 Sperm morphology and chromatin integrity in Swedish warmblood stallions and their relationship to pregnancy rates. Acta Vet Scand 50:2.

Morrell JM \& Rodriguez-Martinez H. 2009a Biomimetic techniques for improving sperm quality in animal breeding: a review. The Open Andrology Journal 1, 1-9. 
Morrell JM, Johannisson A, Strutz, H, Dalin, A-M, Rodriguez-Martinez H. 2009b Colloidal centrifugation of stallion semen: changes in sperm motility, velocity and chromatin integrity during storage. Journal Equine Veterinary Science 29:24-32.

Morrell, J.M.; Johannisson, A.; Dalin, A-M.; Rodriguez-Martinez, H. 2009c Morphology and chromatin integrity of stallion spermatozoa prepared by density gradient and single layer centrifugation through silica colloids. Reprod. Dom. Anim. 44, 512-517.

Morrell JM, Johannisson A, Dalin A-M, Rodriguez-Martinez H 2009d Single Layer Centrifugation with Androcoll ${ }^{\mathrm{TM}}$-E can be scaled-up to allow large volumes of stallion ejaculate to be processed easily. Theriogenology $72,879-884$.

Palmer E, Bezard J, Magistrini M, Duchamp G. 1991. In vitro fertilization in the horse. A retrospective study. J. Reprod. Fertil. Suppl. 44, 375-384.

Roasa LM, Choi YH, Love CC, Romo S, Varner DD, Hinrichs K. 2007. Ejaculate and type of freezing extender affect rates of fertilization of horse oocytes in vitro. Theriogenology 68, 560-566.

Roudebush WE, Worrilow KC, Peters AJ, Witt MA, Toledo AA, Mitchell-Leef D, Carlene W, Massey JB \& Kort HI. Relationship between sperm chromatin integrity, morphology and the hyaluronan binding assay $\left(\mathrm{HBA}^{\mathrm{TM}}\right)$. Proc New England Fertility Soc Annual Meeting 2004.

Thys, M., Vandaele L, Morrell JM, Mestach J, Van Soom A, Hoogewijs M, Rodriguez-Martinez H. (2009) In vitro fertilising capacity of frozen-thawed bull spermatozoa selected by single-layer glycidoxypropyltrimethoxysilane-coated silica colloidal centrifugation. Reproduction in Domestic Animals 44, 390-394.

Worrilow KC, Black N, Maher NB, Bower JB, Peters AJ \& Johnston JB. (2003) Statistically significant correlation between normal sperm morphology (NSM) and sperm binding potential to an immobilized monolayer of hyaluronan using the HYDAK® sperm hyaluronan binding assay (HBA). Proc. ASRM 2003. 
Table 1: cleavage and development of equine oocytes injected with stallion spermatozoa prepared by either Single Layer Centrifugation (SLC) or Density Gradient Centrifugation (DGC).

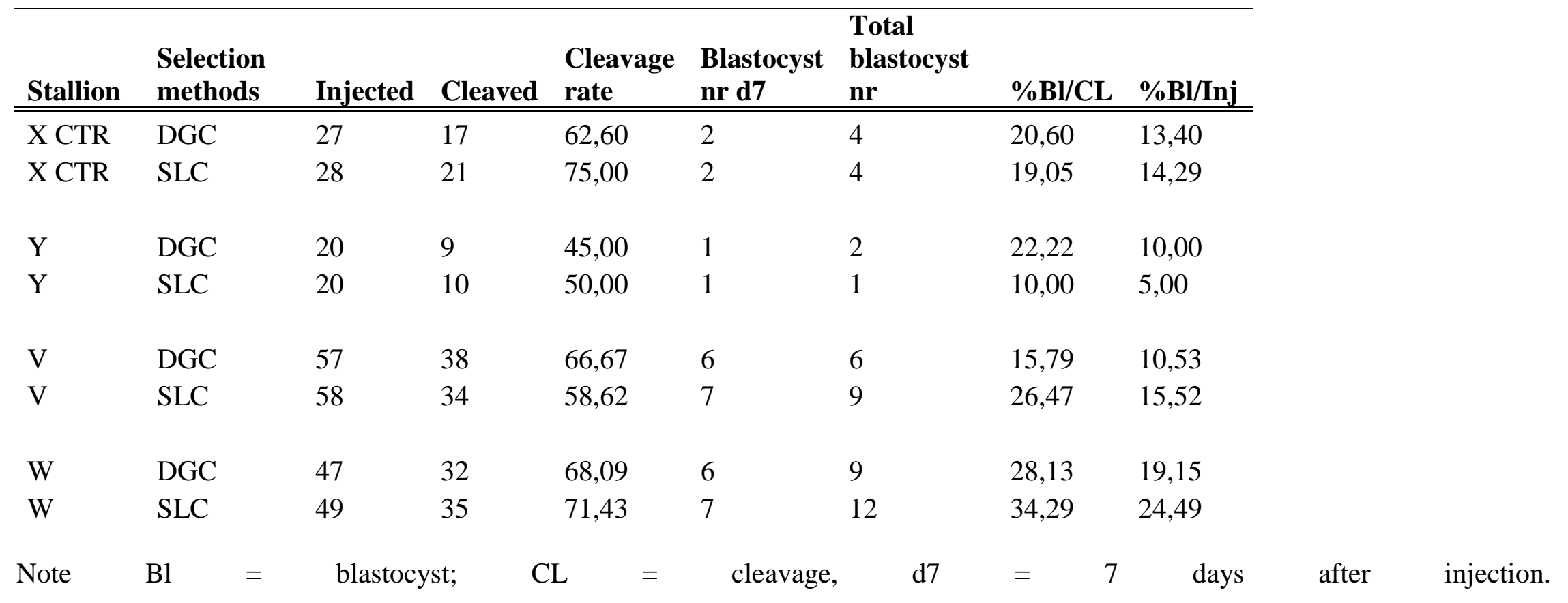


Table 2: proportions (\%) of specific morphological abnormalities in 3 stallion sperm samples prepared by either Single Layer Centrifugation (SLC) or Density Gradient Centrifugation (DGC) ( $n=$ one sample per stallion)

\begin{tabular}{|c|c|c|c|c|c|c|c|c|c|}
\hline & \multicolumn{3}{|c|}{ X (fertile) } & \multicolumn{3}{|c|}{ V (infertile) } & \multicolumn{3}{|c|}{ W (infertile) } \\
\hline & UN & SLC & DGC & UN & SLC & DGC & UN & SLC & DGC \\
\hline $\begin{array}{l}\text { ProxImal } \\
\text { cytoplasmic } \\
\text { droplets }\end{array}$ & 2,5 & 0 & 0 & 10,5 & 3 & 2,5 & 1 & 1 & 3 \\
\hline heads & 11,4 & 18 & 9,4 & 31,8 & 34,4 & 37,8 & 11,8 & 12,4 & 24.8 \\
\hline $\begin{array}{l}\text { Midpiece } \\
\text { defect } \\
\text { nuclear }\end{array}$ & 6 & 6,5 & 2,5 & 36,5 & 12,5 & 29 & 3 & 5 & 11,5 \\
\hline vacuoles & 1 & 0,5 & 1,5 & 2 & 1 & 6 & 0 & 1,5 & 3,5 \\
\hline tails & 4 & 6 & 0,3 & 13,8 & 27 & 8 & 1,8 & 2,8 & 1,8 \\
\hline
\end{tabular}

Note: $\mathrm{UN}=$ uncentrifuged, $\mathrm{SLC}=$ Single layer centrifugation; DGC $=$ density gradient centrifugation. Since the sperm concentration was very low in the SLC and DGC samples from the infertile stallions ( $\mathrm{V}$ and $\mathrm{W}$ ), the smears contained very few spermatozoa and the morphological evaluation was done on only 100 spermatozoa instead of 200 or 500 . Therefore, the count for these samples may be inaccurate compared to the uncentrifuged samples from the same stallion. 
Table 3. Development to blastocyst stage of equine oocytes injected with SLC or SLC-PICSI HA selected sperm

\begin{tabular}{|c|c|c|c|c|c|c|c|c|}
\hline $\begin{array}{l}\text { Sperm } \\
\text { treatment }\end{array}$ & Stallion & Inj. & Cleaved & $\begin{array}{l}\text { Cleavage } \\
\text { rate }\end{array}$ & Blast. d7 & $\begin{array}{l}\text { Total } \\
\text { blast. }\end{array}$ & $\% \mathrm{Bl} / \mathrm{CL}$ & $\%$ Bl/Inj \\
\hline CTR & CTR & 28 & 19 & 67,86 & 2 & 4 & 21,05 & 14,29 \\
\hline CTR & CTR 2 & 16 & 10 & 62,50 & 2 & 2 & 20,00 & 12,50 \\
\hline CTR & CTR & 20 & 15 & 75,00 & 3 & 4 & 26,67 & 20,00 \\
\hline CTR & W & 10 & 6 & 60,00 & 0 & 1 & 16,67 & 10,00 \\
\hline \multirow[t]{2}{*}{ CTR } & CTR 2 & 20 & 16 & 80,00 & 0 & 2 & 12,50 & 10,00 \\
\hline & & 94 & 66 & $70,21^{a}$ & 7 & 13 & $19,70^{a}$ & $13,83^{a}$ \\
\hline PICSI & CTR & 20 & 8 & 40,00 & 2 & 3 & 37,50 & 15,00 \\
\hline PICSI & CTR 2 & 22 & 9 & 40,91 & 2 & 3 & 33,33 & 13,64 \\
\hline PICSI & CTR & 20 & 15 & 75,00 & 2 & 4 & 26,67 & 20,00 \\
\hline PICSI & $\mathrm{W}$ & 10 & 3 & 30,00 & 1 & 1 & 33,33 & 10,00 \\
\hline \multirow[t]{2}{*}{ PICSI } & CTR 2 & 20 & 8 & 40,00 & 3 & 4 & 50,00 & 20,00 \\
\hline & & 92 & 43 & $46,74^{b}$ & 10 & 15 & $34,88^{b}$ & $16,30^{a}$ \\
\hline
\end{tabular}

Student $T$ test. Superscripts denote statistical significance between columns $(p<0,05)$ 
Figure 1: chromatin damage (DFI \%) in stallion spermatozoa prepared for ICSI by Single Layer Centrifugation or Density Gradient Centrifugation (one samples for each stallion and treatment).

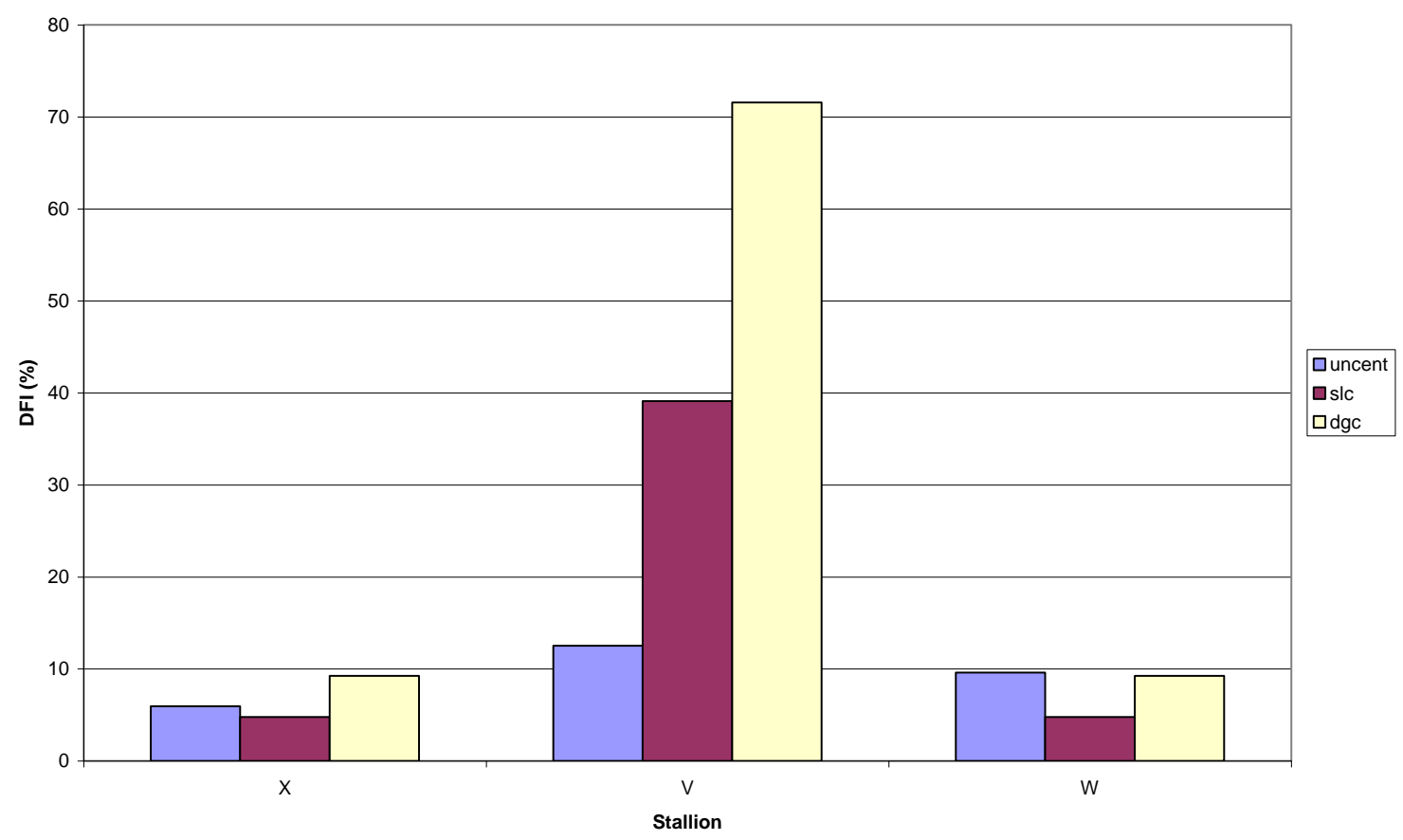

$\mathrm{UN}=$ uncentrifuged, SLC = Single layer centrifugation; $\mathrm{DGC}=$ density gradient centrifugation. Stallion $\mathrm{X}=$ fertile; stallions $\mathrm{V}$ and $\mathrm{W}=$ infertile. 
Figure 2: Binding of stallion spermatozoa to hyaluronan-coated slides $(n=11)$.

Differences in binding of stallion sperm to hyaluronan after cold storage for $24 \mathrm{~h}(\mathrm{n}=11)$

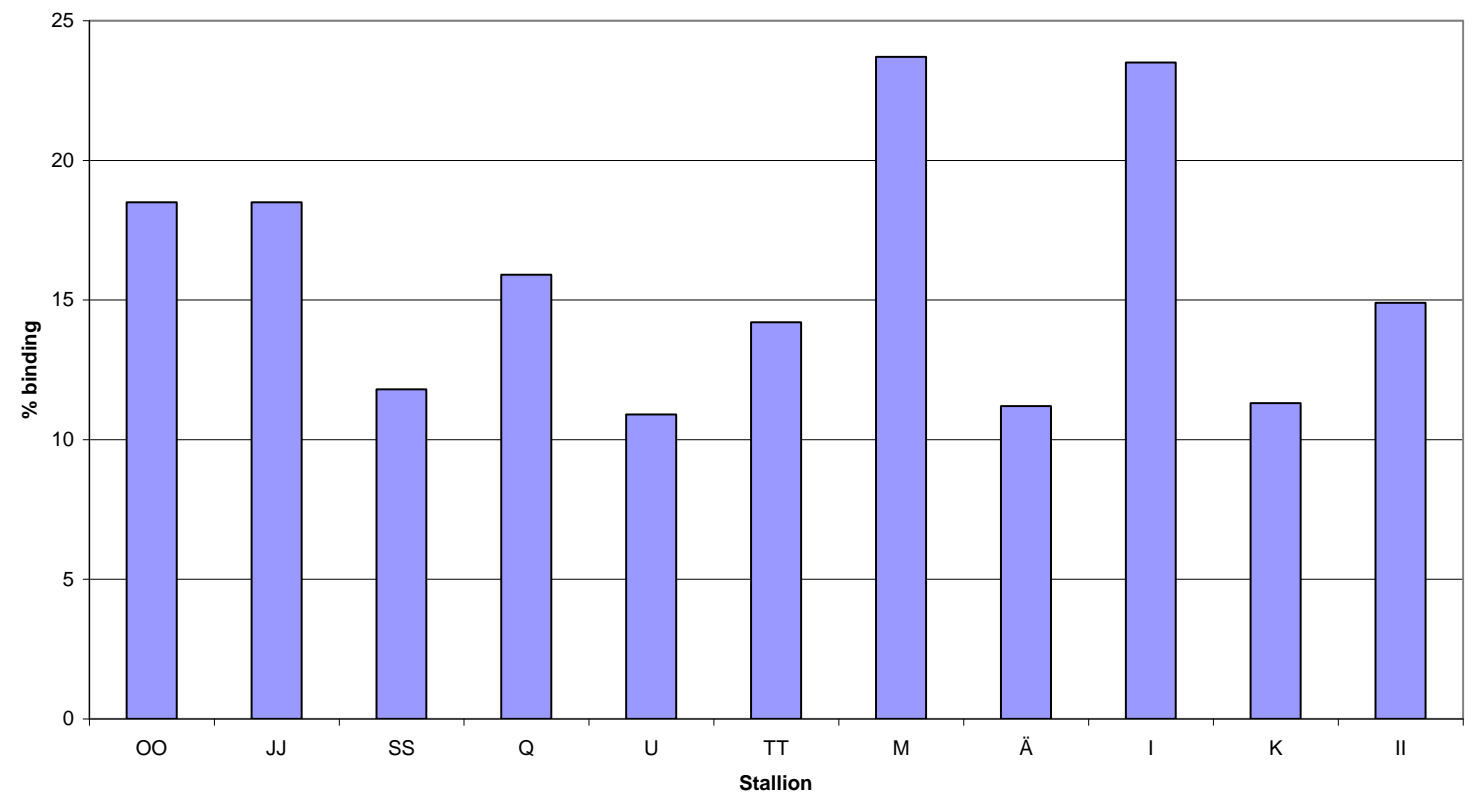

